\title{
Benchmarking Electron Densities and Electrostatic Potentials of Proteins from the Three-Partition Frozen Density Embedding Method
}

\section{- Supporting Information -}

\author{
Albrecht Goez and Johannes Neugebauer ${ }^{1}$ \\ Theoretische Organische Chemie, Organisch-Chemisches Institut \\ and Center for Multiscale Theory and Computation, \\ Westfälische Wilhelms-Universität Münster \\ Corrensstraße 40, 48149 Münster, Germany
}

Date: $\quad$ August 25, 2016

Status: $\quad$ Revised version, re-submitted to J. Chem. Theory Comput.

\footnotetext{
${ }^{1}$ email: j.neugebauer@uni-muenster.de
} 


\section{ESP Differences due to Basis Set and Method}

Tab. S1 lists MADs in the ESP obtained for some test systems with different combinations of methods and basis sets. While panel (a) gives differences between MP2 and various HF/DFT methods obtained with the same basis set (cc-pVDZ), the values in panel (b) represent the differences between the STO basis set DZP and the GTO basis set cc-pVDZ obtained with the same method (first column). It can be seen that similar to the density (see Tab. I in the main article), the basis set deviations are about twice as large as those due to the method. This confirms that direct comparisons between GTO and STO results would be significantly obscured.

\section{Supermolecular Results for TC5b with Different Functionals}

Fig. S1 gives errors for the electron density and the ESP obtained in supermolecular calculations with different XC functionals and HF for TC5b in solution. The reference are M06-2X calculations for the density and CAMY-B3LYP results for the ESP.

\section{Density Errors for GGA and Hybrid Functionals with Different Fragmentation Sizes}

Fig. S2 demonstrates the density convergence behavior for TC5b in solution with different combinations of method and fragment size. For all pure density functionals (PBE, BP86, BLYP), the results after three FT cycles are independent of the fragmentation size. In case of a hybrid functional (B3LYP, PBE0, CAMY-B3LYP), however, distinct results are 
Table S1: MADs in the ESP for different test systems in solution. (a) Differences between MP2 and various methods (first column) obtained with the same basis set (cc-pVDZ). (b) Differences between DZP and cc-pVDZ basis sets obtained with the same method (first column). All values in atomic units.

(a) Method/cc-pVDZ - MP2/cc-pVDZ

\begin{tabular}{lccccccc} 
Method & $(\text { Gly })_{2}$ & $(\text { Gly })_{5}$ & $(\text { Gly })_{10}$ & $(\text { Gly })_{20}$ & TC5b & IGF-F1-1 & RCB \\
\hline BLYP & 0.0018 & 0.0012 & 0.0010 & 0.0009 & 0.0010 & 0.0011 & 0.0009 \\
B3LYP & 0.0006 & 0.0007 & 0.0008 & 0.0008 & 0.0012 & 0.0013 & 0.0007 \\
M06-2X & 0.0011 & 0.0010 & 0.0010 & 0.0010 & 0.0015 & 0.0014 & 0.0010 \\
CAM-B3LYP & 0.0008 & 0.0009 & 0.0009 & 0.0010 & 0.0015 & 0.0015 & 0.0008 \\
HF & 0.0042 & 0.0038 & 0.0035 & 0.0033 & 0.0039 & 0.0040 & 0.0025
\end{tabular}

(b) Method/DZP - Method/cc-pVDZ

\begin{tabular}{lccccccc} 
Method & $(\text { Gly })_{2}$ & Gly $_{5}$ & $(\text { Gly })_{10}$ & $(\text { Gly })_{20}$ & TC5b & IGF-F1-1 & RCB \\
\hline BLYP & 0.0032 & 0.0030 & 0.0030 & 0.0029 & 0.0040 & 0.0042 & 0.0035 \\
B3LYP & 0.0031 & 0.0030 & 0.0030 & 0.0030 & 0.0040 & 0.0042 & 0.0035 \\
M06-2X & 0.0033 & 0.0033 & 0.0033 & 0.0032 & 0.0041 & 0.0043 & 0.0037 \\
CAM-B3LYP $^{a}$ & 0.0030 & 0.0030 & 0.0030 & 0.0030 & 0.0039 & 0.0041 & 0.0035 \\
HF & 0.0033 & 0.0034 & 0.0035 & 0.0036 & 0.0043 & 0.0046 & 0.0038
\end{tabular}

${ }^{a}$ Comparison between CAM-B3LYP/cc-pVDZ and CAMY-B3LYP/DZP 
(a) Density

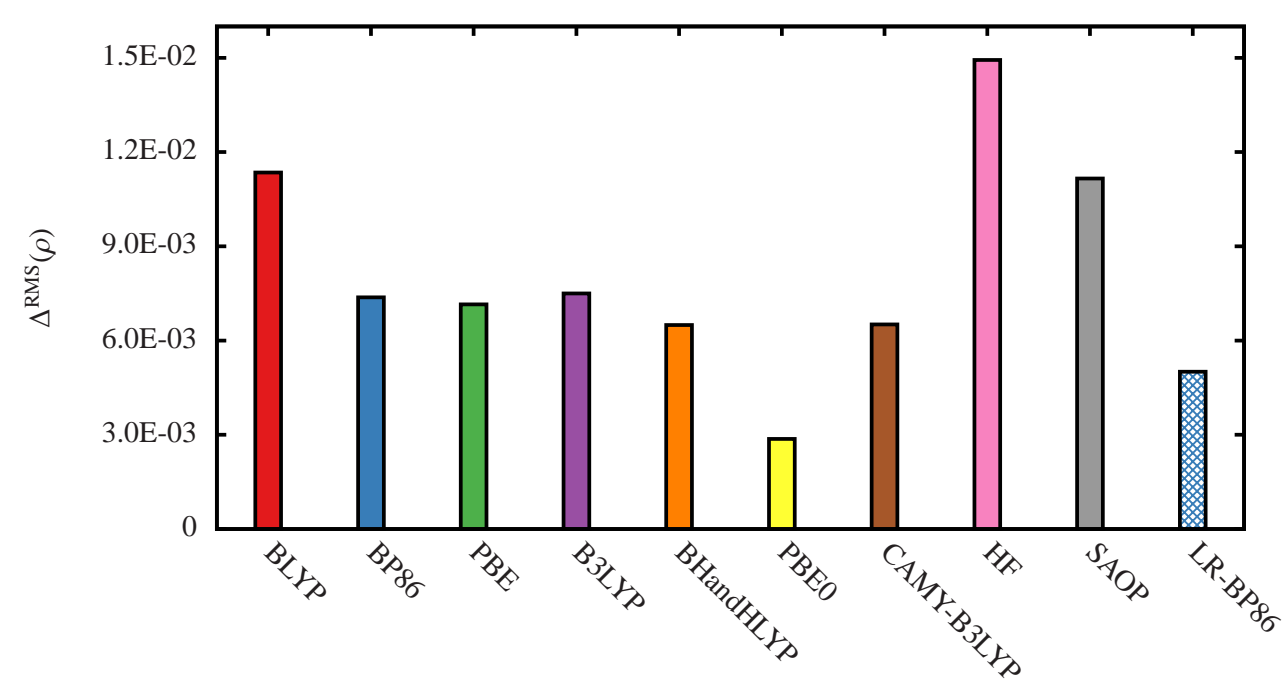

Functional

(b) ESP

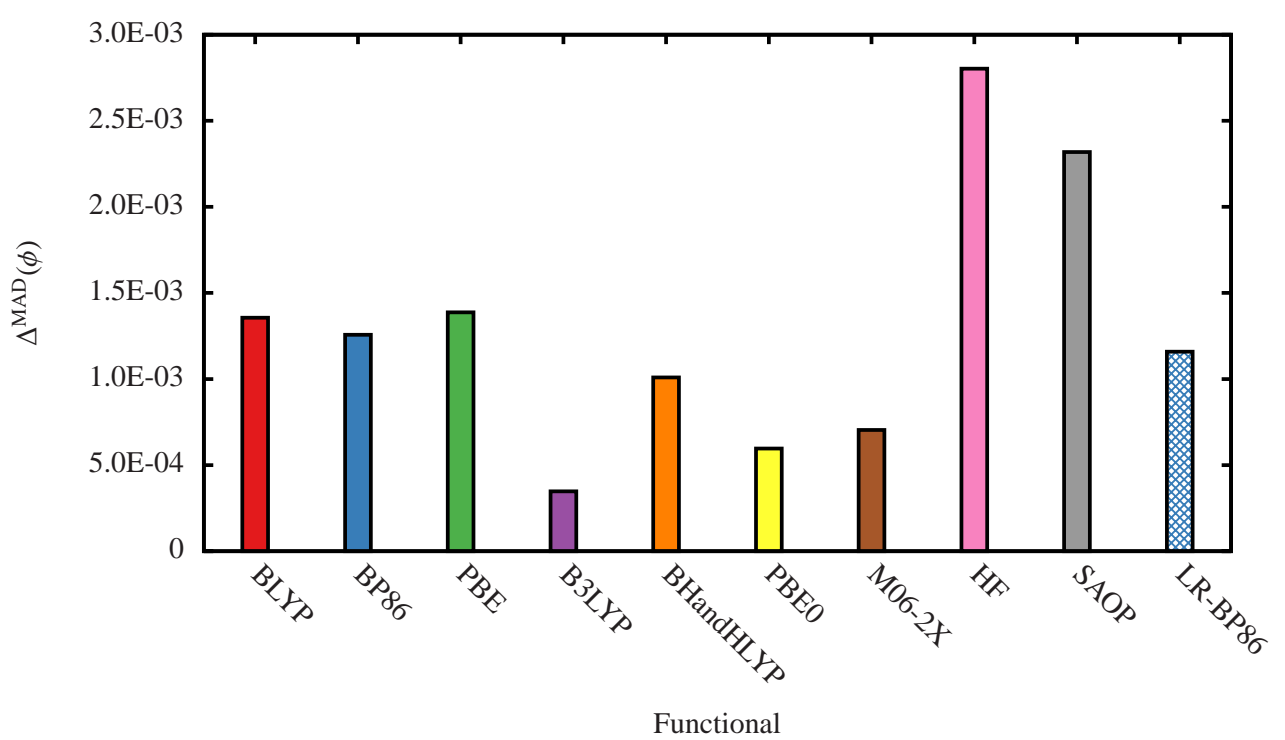

Figure S1: Influence of the XC functional on density and ESP errors in supermolecular calculations for TC5b in solution (STO basis set: DZP). Errors are given with respect to supermolecular results obtained with M06-2X (density) or CAMY-B3LYP (ESP). 
obtained, which must be attributed to the non-additive $\mathrm{XC}$ contribution to the embedding potential (see Sec. 2 in the main article).

\section{Effect of the Cap Convergence Threshold}

Fig. S3 demonstrates the effect of the cap density convergence threshold on the density and ESP errors for TC5b in solution. It is clear that the effect of varying the threshold is almost negligible. Interestingly, a stricter value even leads to slightly larger errors, even though the absolute differences are tiny. It is thus confirmed that the quality of the results is invariant with respect to the threshold (within the tested range) and the latter can rather be chosen in order to secure robust convergence (see Sec. 2 in the main article for details).

\section{$5 \quad$ Results for Other Proteins}

In addition to TC5b, the miniproteins IGF-F1-1 and RCB-1 were investigated. The effect of the most important parameters (XC functional and fragmentation size) on density and ESP errors is represented in Figs. S4 and S5.

The behavior for the additional test systems is qualitatively similar to TC5b (see Sec. 5 in the main article), especially with respect to the convergence over the course of the FT cycles. Between the second and third cycle, both properties remain essentially unchanged.

Concerning the $\mathrm{XC}$ functional, the converged results are again of similar accuracy as supermolecular calculations with the same functional (see Sec. 5.1 in the main article and Fig. S1 for TC5b results). While the tested hybrid functionals in general yield a better representation of the ESP, the total density error is smaller with PBE than with B3LYP. 
(a) PBE

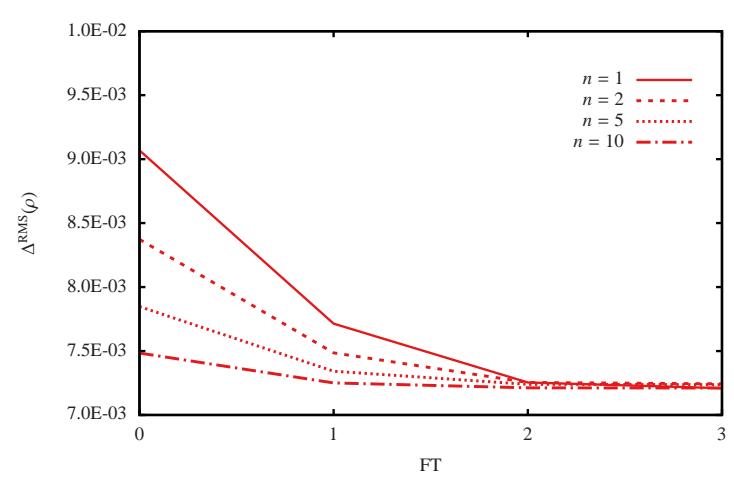

(c) BLYP

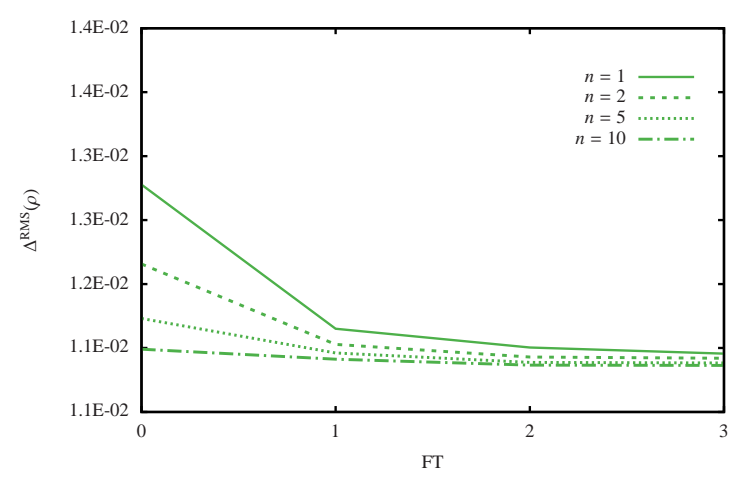

(e) PBE0

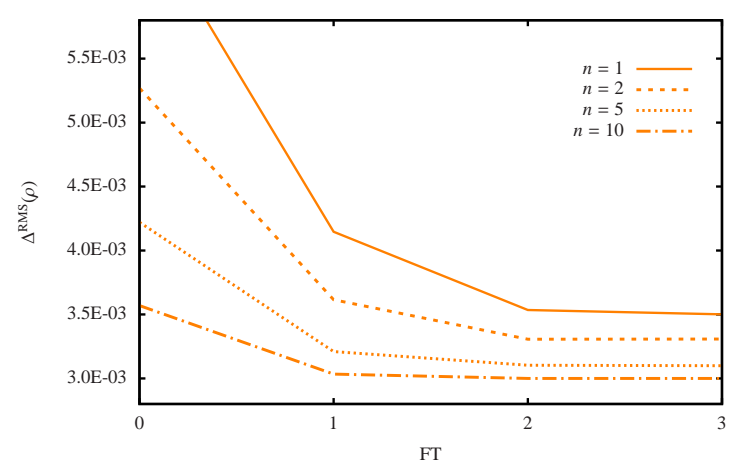

(b) BP86

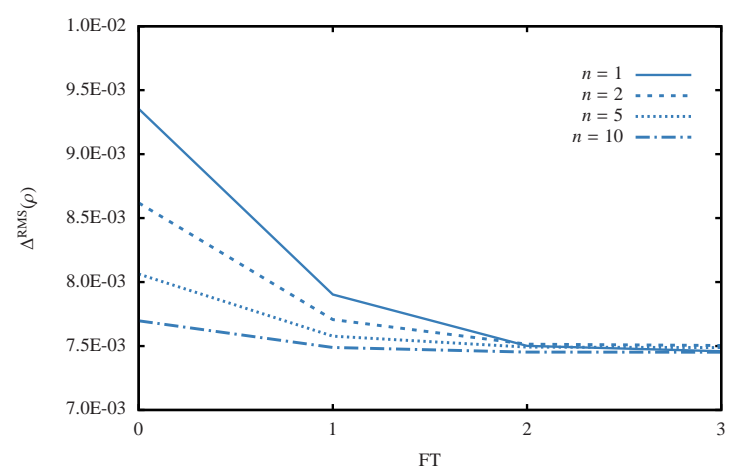

(d) B3LYP

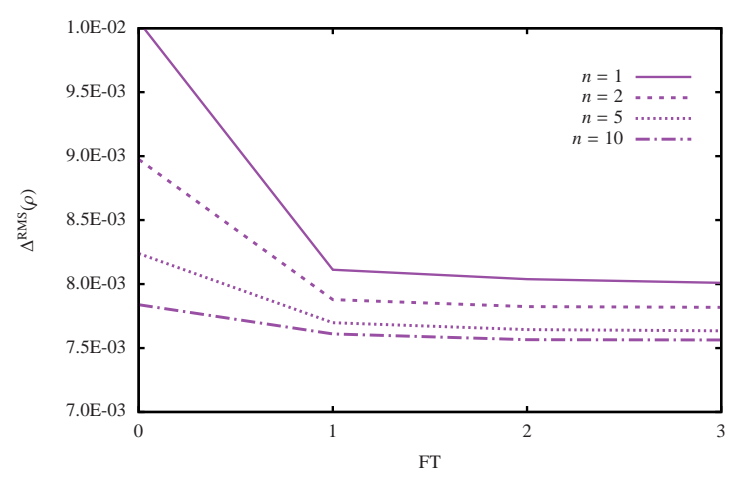

(f) CAMY-B3LYP

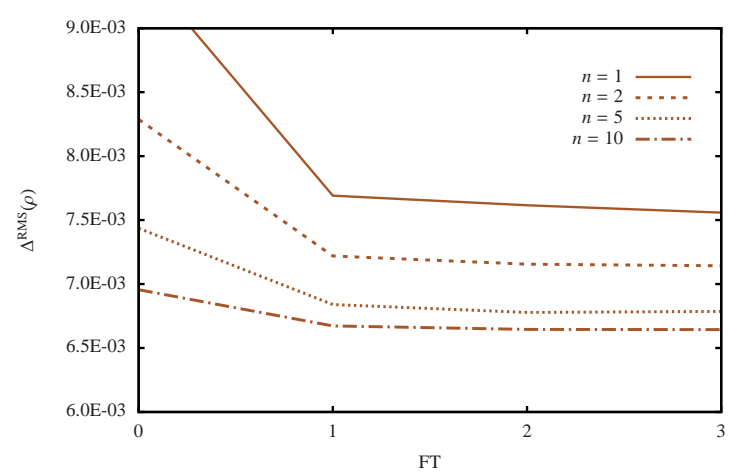

Figure S2: Influence of the fragment size on the density errors in 3-FDE calculations for TC5b in solution over the course of three FT cycles (cycle $0=$ MFCC) with different $\mathrm{XC}$ functionals. Errors are given with respect to supermolecular results obtained with M06-2X. The scale of the $y$ axis is identical in all panels. 
(a) Density

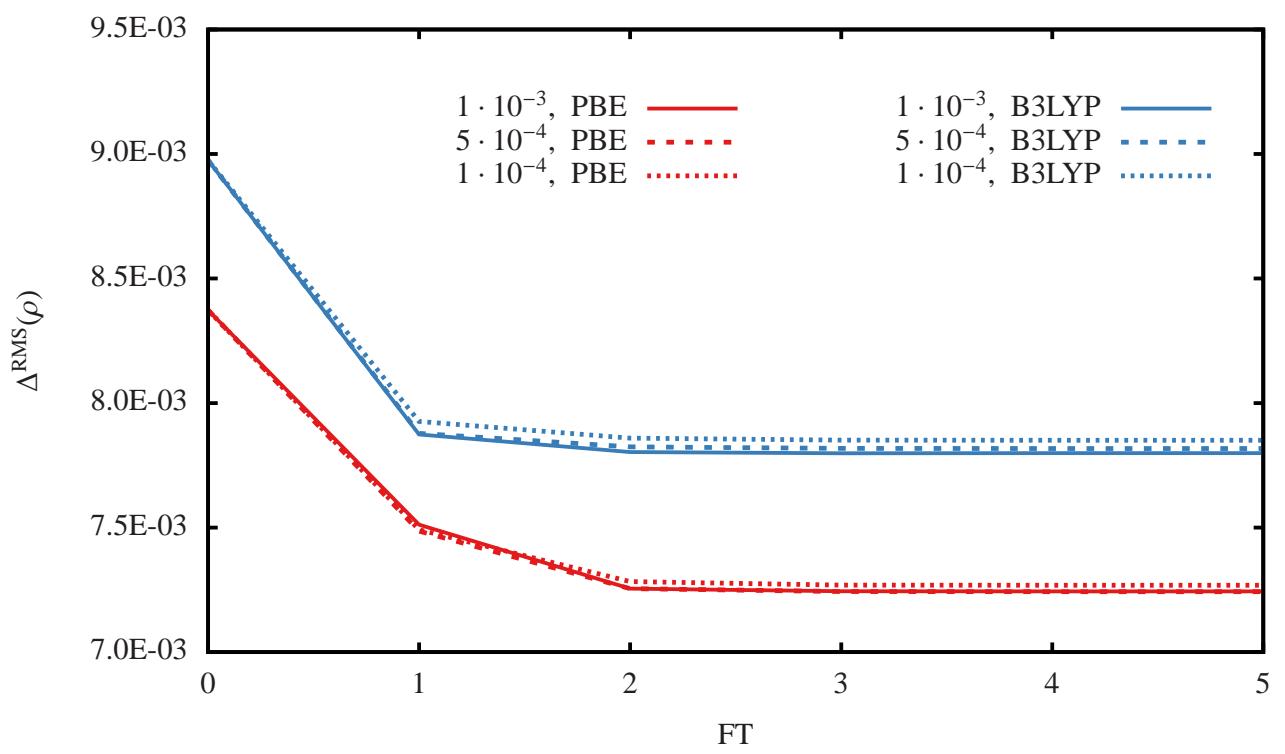

(b) ESP

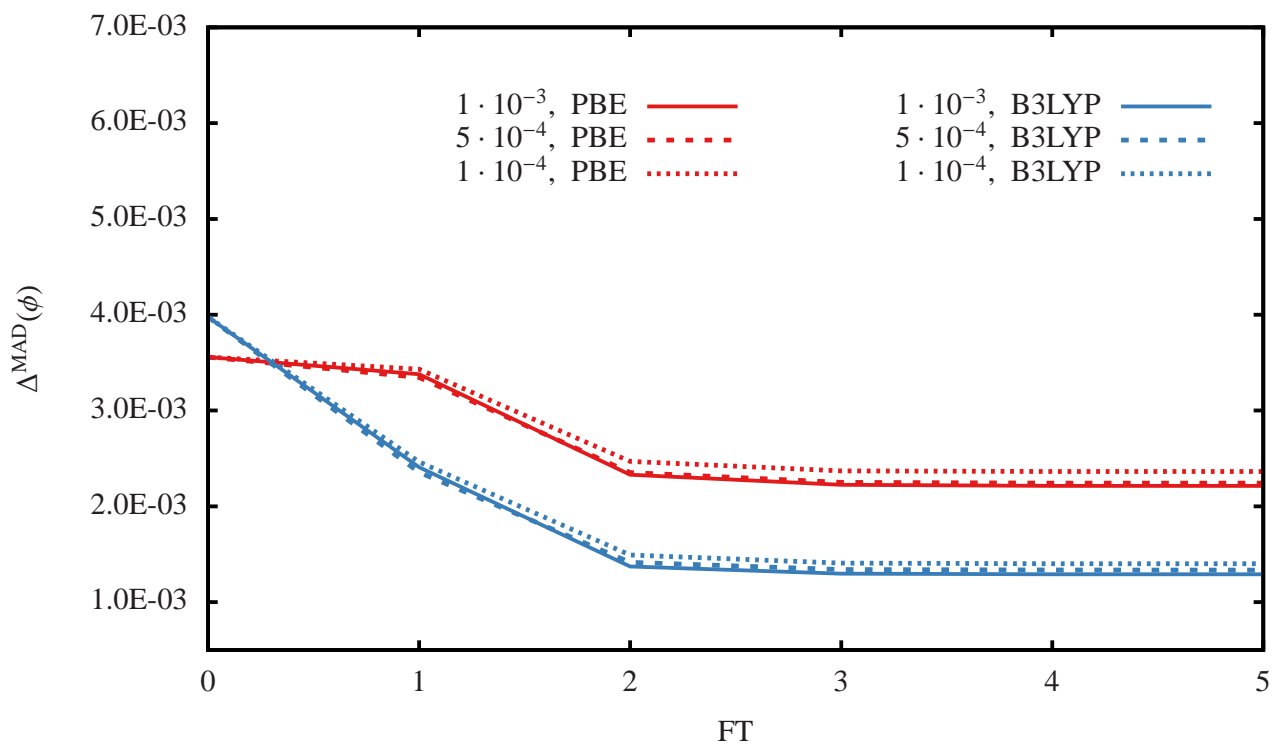

Figure S3: Influence of the cap convergence threshold on density and ESP errors in 3-FDE calculations for TC5b in solution over the course of five FT cycles (cycle $0=$ MFCC). Errors are given with respect to supermolecular results obtained with M06-2X (density) or CAMY-B3LYP (ESP). 
(a) Density

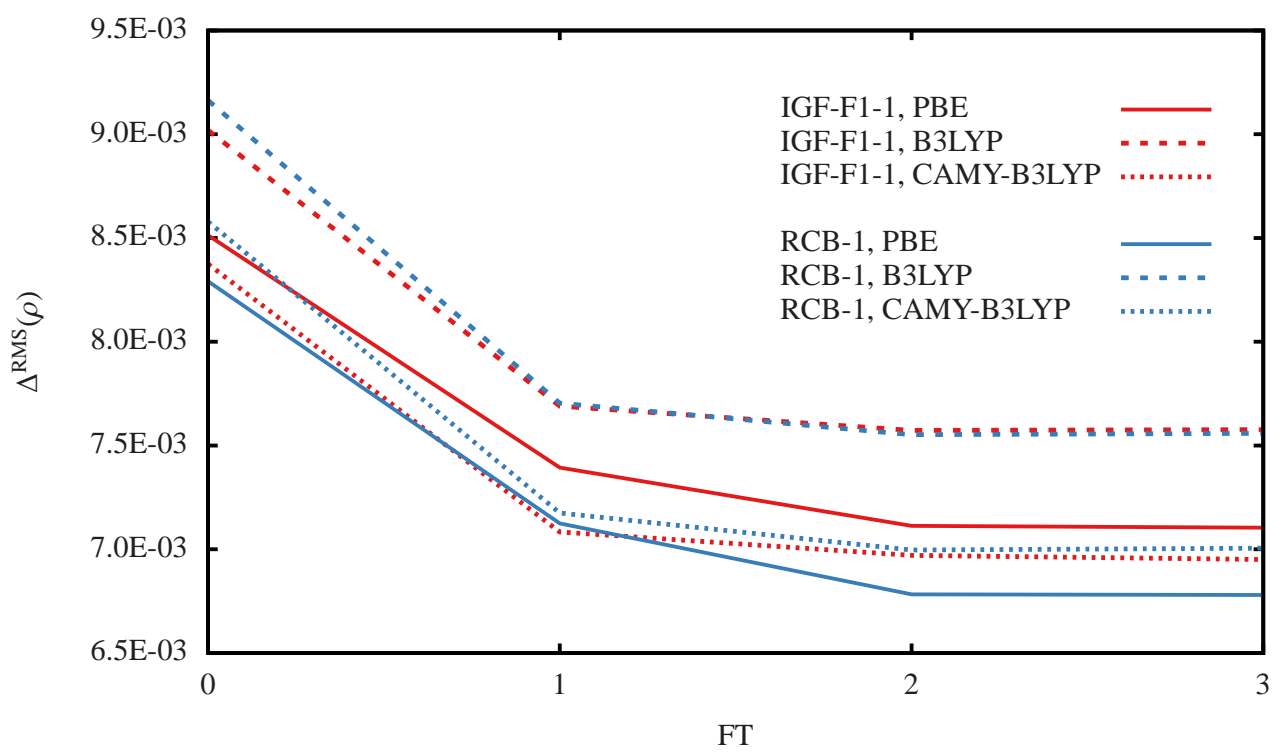

(b) ESP

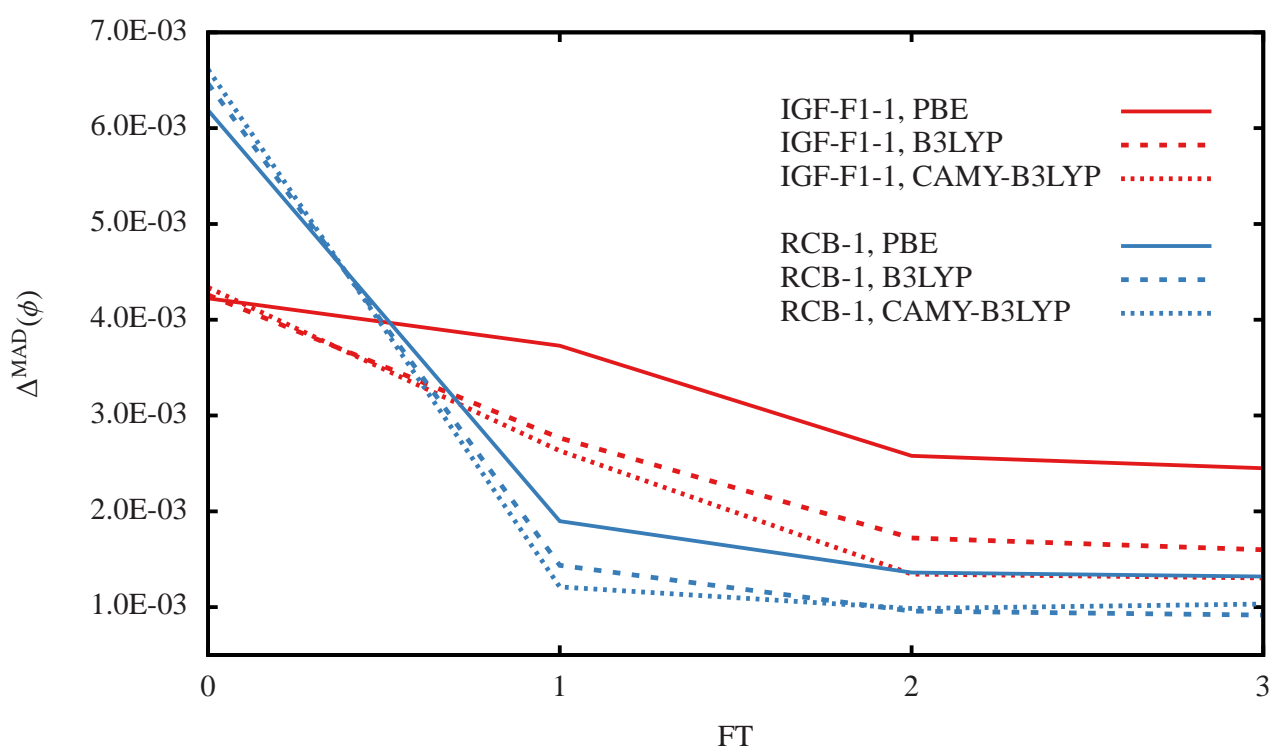

Figure S4: Influence of the XC functional on density and ESP errors in 3-FDE calculations for IGF-F1-1 and RCB-1 in solution over the course of three FT cycles (cycle $0=$ MFCC). Errors are given with respect to supermolecular results obtained with M06-2X (density) or CAMY-B3LYP (ESP). 
(a) Density

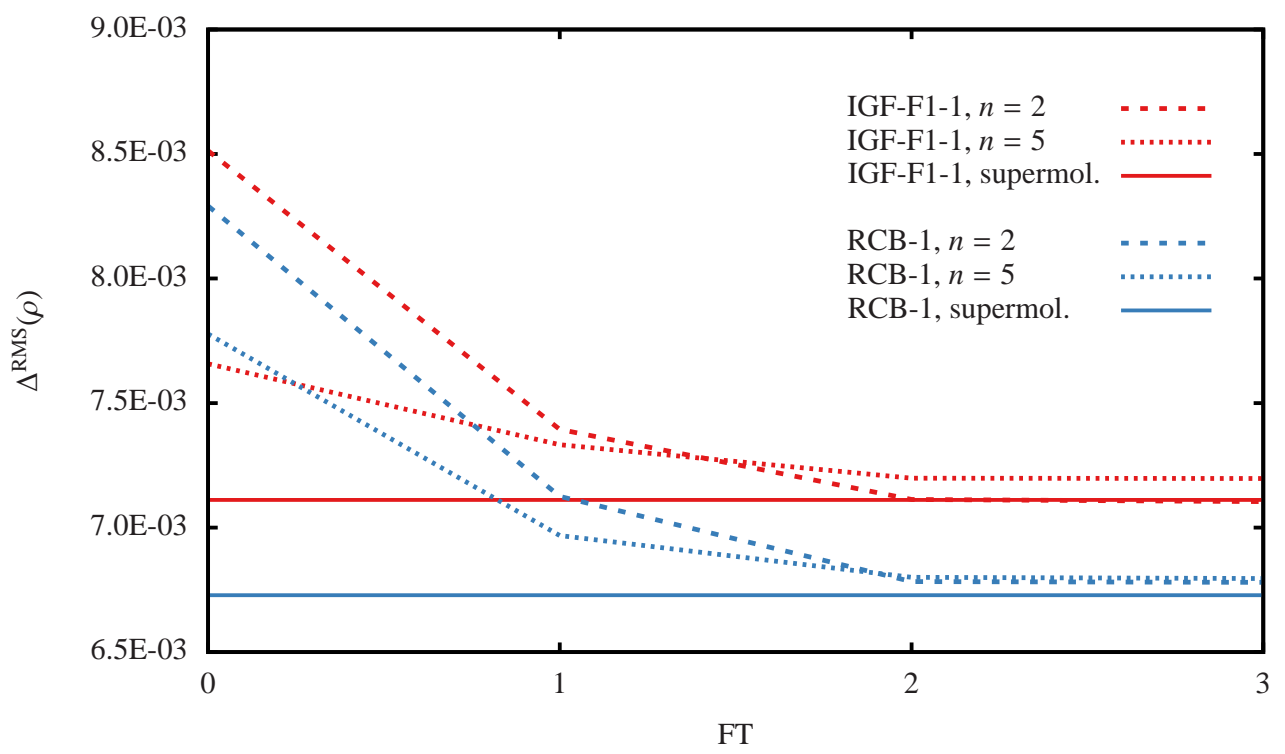

(b) ESP

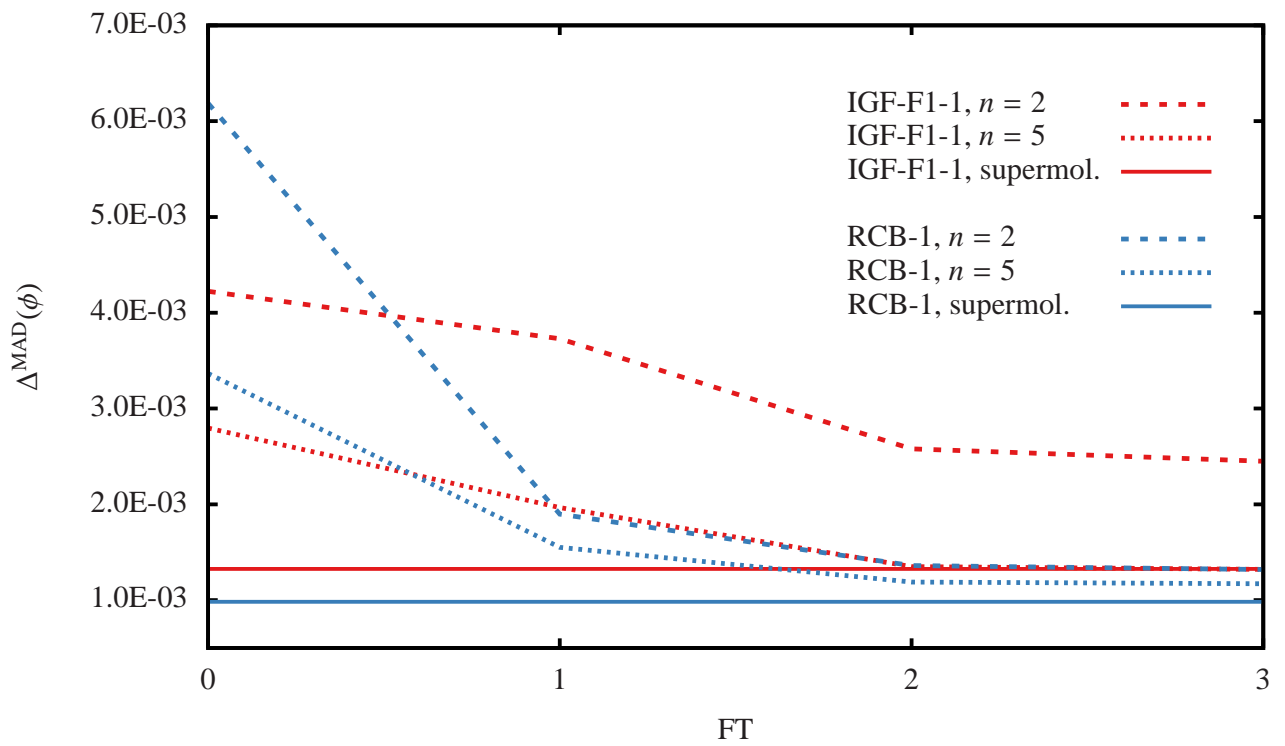

Figure S5: Influence of the fragment size on density and ESP errors in 3-FDE calculations for IGF-F1-1 and RCB-1 in solution over the course of three FT cycles (cycle $0=$ MFCC). Errors are given with respect to supermolecular results obtained with M06-2X (density) or CAMY-B3LYP (ESP). 
Similar errors are obtained for both proteins.

The choice of fragmentation pattern also has the same implications for all tested proteins (Fig. S5). Systematic convergence is observed over the course of two FT cycles and for the present case (PBE functional), all fragment sizes eventually lead to the same deviation (see Fig. S2 for the effect of hybrid functionals).

\section{Influence of the Cap Radius}

As mentioned in Sec. 2, the cap region is defined by all points of the numerical integration grid which are within a certain radius of a cap atom and not closer to any non-cap atom. In order to make sure that the default value of $3 a_{0}$ is suitable, a series of tests for the RCB-1 miniprotein was carried out. This structure was specifically chosen because it also includes capped disulfide bridges (featuring large sulfur cap atoms). Fig. S6 shows the influence of this parameter for different choices over the course of three sequential FT cycles with a fragment size of $n=5$ and the PBE functional.

It is evident that the results are hardly affected by this choice (note that the scales are identical to Figs. S4 and S5). Incidentally, the default value of $r=3.0 a_{0}$ performs best for both density and ESP.

\section{$7 \quad$ Influence of the Basis Set}

In order to make sure that the results presented in the main part of this article are not a consequence of the relatively small DZP basis set (double- $\zeta$ with one set of polarization functions), the density and ESP errors for TC5b with the PBE functional and various fragmentation sizes were also determined with the much larger TZ2P set (triple- $\zeta$ with 
(a) Density

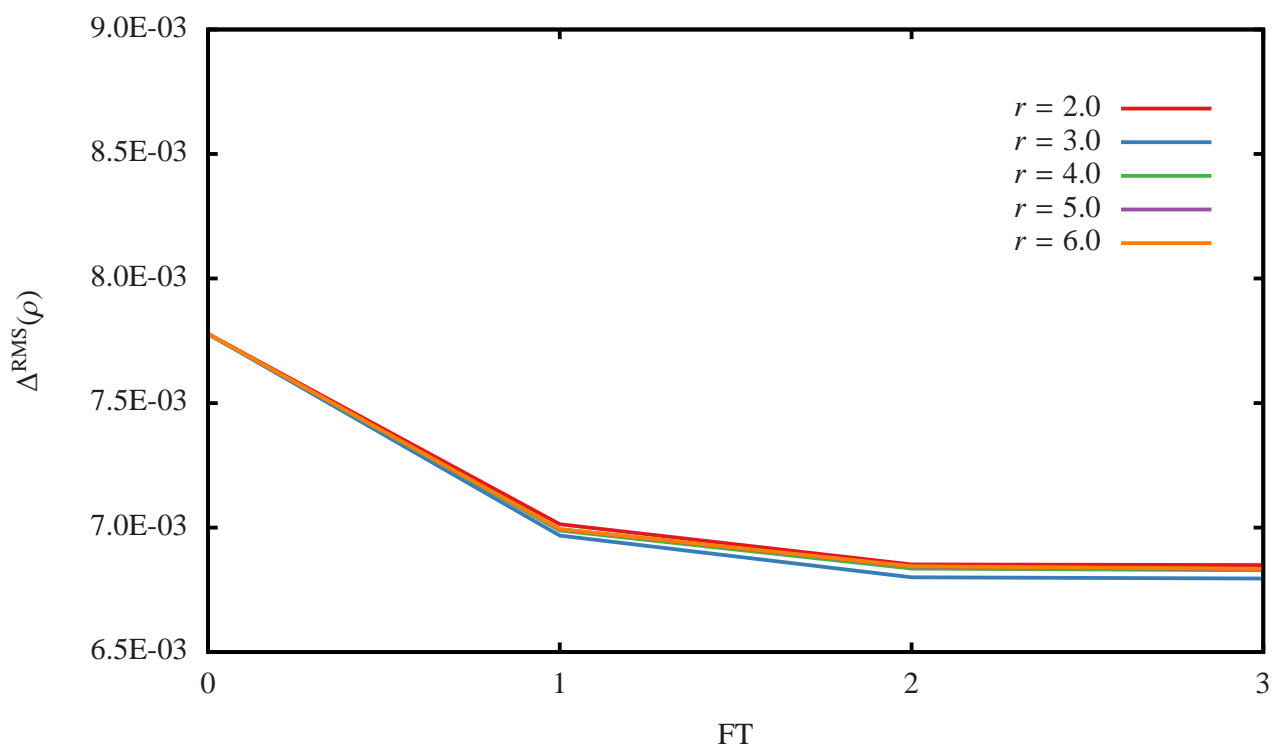

(b) ESP

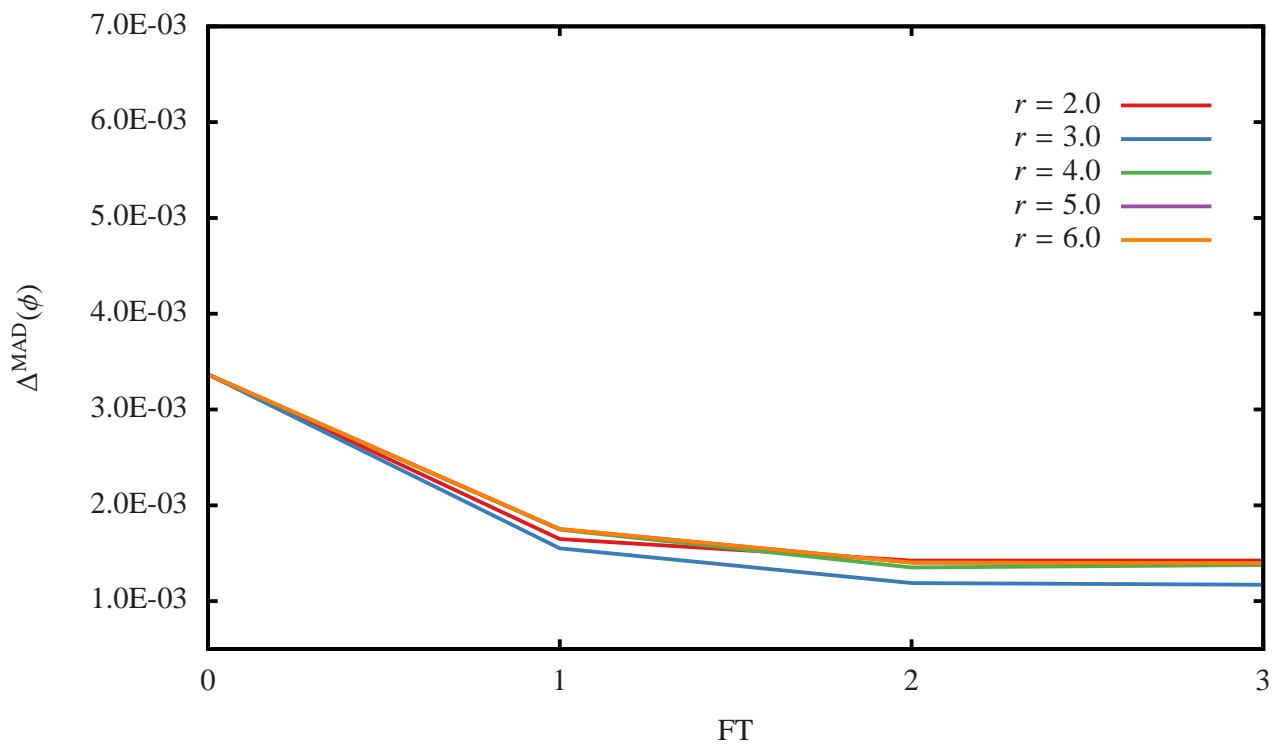

Figure S6: Influence of the cap radius on density and ESP errors in 3-FDE calculations for RCB-1 in solution over the course of three FT cycles (cycle $0=\mathrm{MFCC}$ ). Errors are given with respect to supermolecular results obtained with M06-2X (density) or CAMY-B3LYP (ESP). 
two sets of polarization functions). Of course, also the reference data were obtained with the larger set in that case. Both the DZP and TZ2P results are presented in Fig. S7.

It can be seen that the two basis sets yield qualitatively very similar results. In case of the density, differences are mainly evident for the MFCC results and decrease with the number of FT cycles. For the ESP, the TZ2P curves generally display slightly smoother behavior than their DZP counterparts, but especially for the converged results (after three FT cycles) identical qualitative features are again observed.

\section{Influence of the Capping Group Type}

In this section, the effect of the nature of the capping groups used for saturating the individual fragments is investigated. Four different pairs of capping groups were tested:

- Single hydrogen atoms

- Neutral amino acid termini $\left(\mathrm{COH}\right.$ and $\left.\mathrm{NH}_{2}\right)$ - "Small"

- Methylated amino acid termini $\left(\mathrm{COCH}_{3}\right.$ and $\left.\mathrm{NHCH}_{3}\right)$ - "Regular"

- Whole next amino acid on both sides capped with a neutral terminus - "Large"

It should be noted that for the "Large" scheme, one of the neighboring residues had to be neutralized to be used as a capping group, which is due to problems with the implementation for charged capping groups.

In case of capping with hydrogen atoms, none of the fragments could be converged. This is most likely due to the distinctly different electronic situation between the resulting $\mathrm{C}-\mathrm{H}$ or $\mathrm{N}-\mathrm{H}$ bond and the real system, which contains a peptide bond in the same place. 
(a) Density

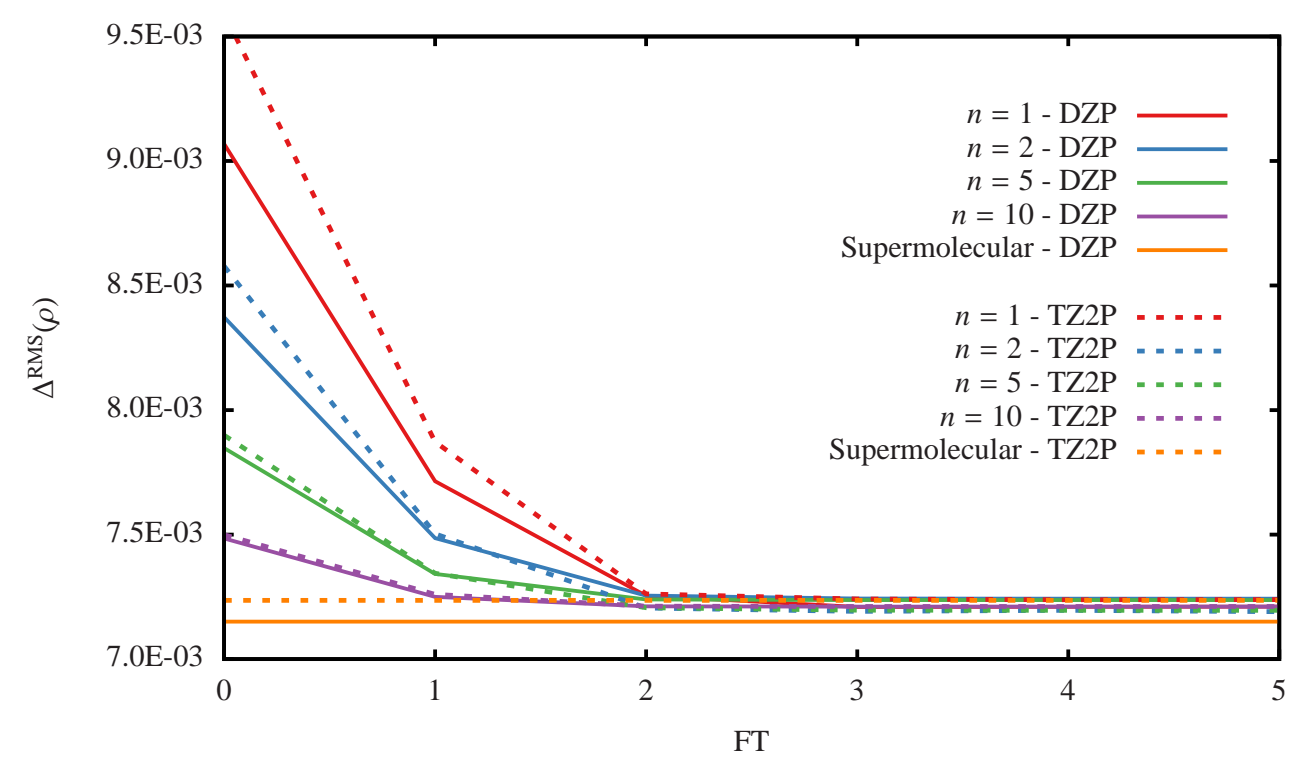

(b) ESP

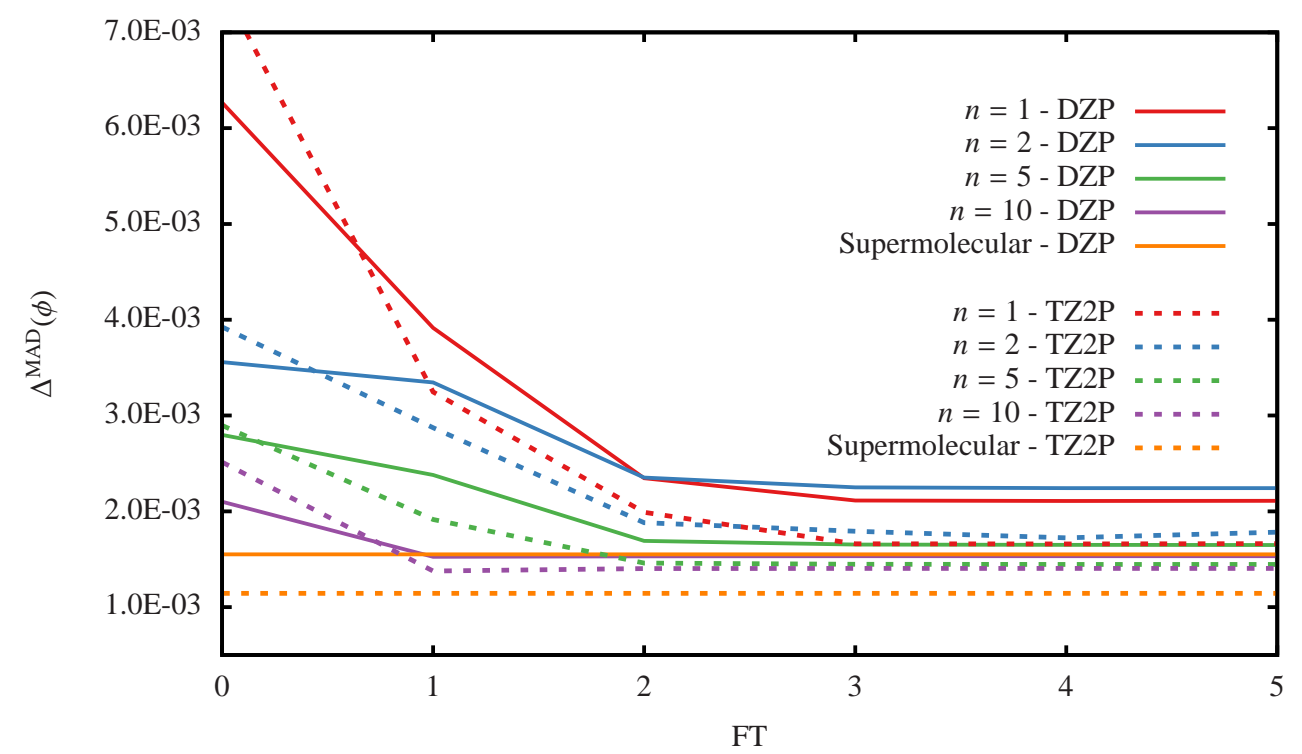

Figure S7: Influence of fragment size and basis set on density and ESP errors in 3-FDE calculations for TC5b in solution over the course of five FT cycles (cycle $0=$ MFCC). Errors are given with respect to supermolecular results obtained with M06-2X (density) or CAMY-B3LYP (ESP). 
The density and ESP errors obtained for the other three capping schemes with the PBE functional and a fragment size of $n=5$ are shown for TC5b in Fig. S8.

The results for the "Small" and "Regular" capping groups are almost indistinguishable. Interestingly, in case of the "Large" version increased errors are observed for the MFCC results and the first FT cycle, whereas smaller deviations are obtained for all further relaxation steps. While the absolute values are almost identical for the density, a notably smaller deviation is obtained for the converged ESP. Depending on the number of FT cycles to be carried out, larger capping groups thus offer a possibility to further reduce the density error. However, the increased computational effort connected with the much larger fragments does not in general justify the small improvements observed here.

\section{Influence of COSMO Cavity Parameters}

The tests presented in Sec. 4 were carried out with the default settings for the construction of the COSMO cavity in all cases. However, while the construction algorithm as well as the default values for the atomic radii are identical in both TURBOMOLE 7.0 and OrCA 3.0.3, they differ slightly in ADF 2014.01. To make sure that this does not significantly affect the presented results, the density and ESP differences between DZP results with different functionals $(\mathrm{ADF})$ and cc-pVDZ results with the same method (ORCA) have been recalculated with the adapted settings for ADF. The results are presented in Tab. S2.

The effect is clearly negligible for the density, and also the ESP is only mildly affected. The deviations in the ESP are still more than twice as large as those with respect to MP2/cc-pVDZ results (see Tab. S1 (a)). 
(a) Density

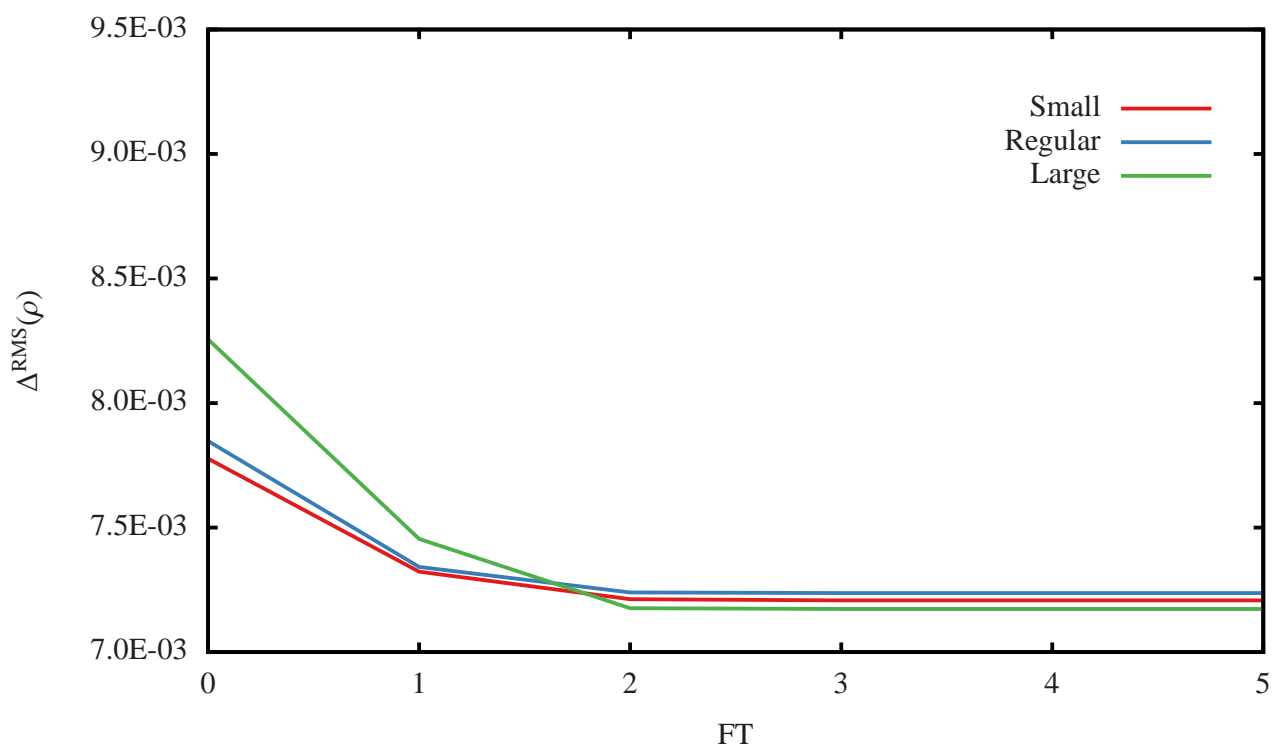

(b) ESP

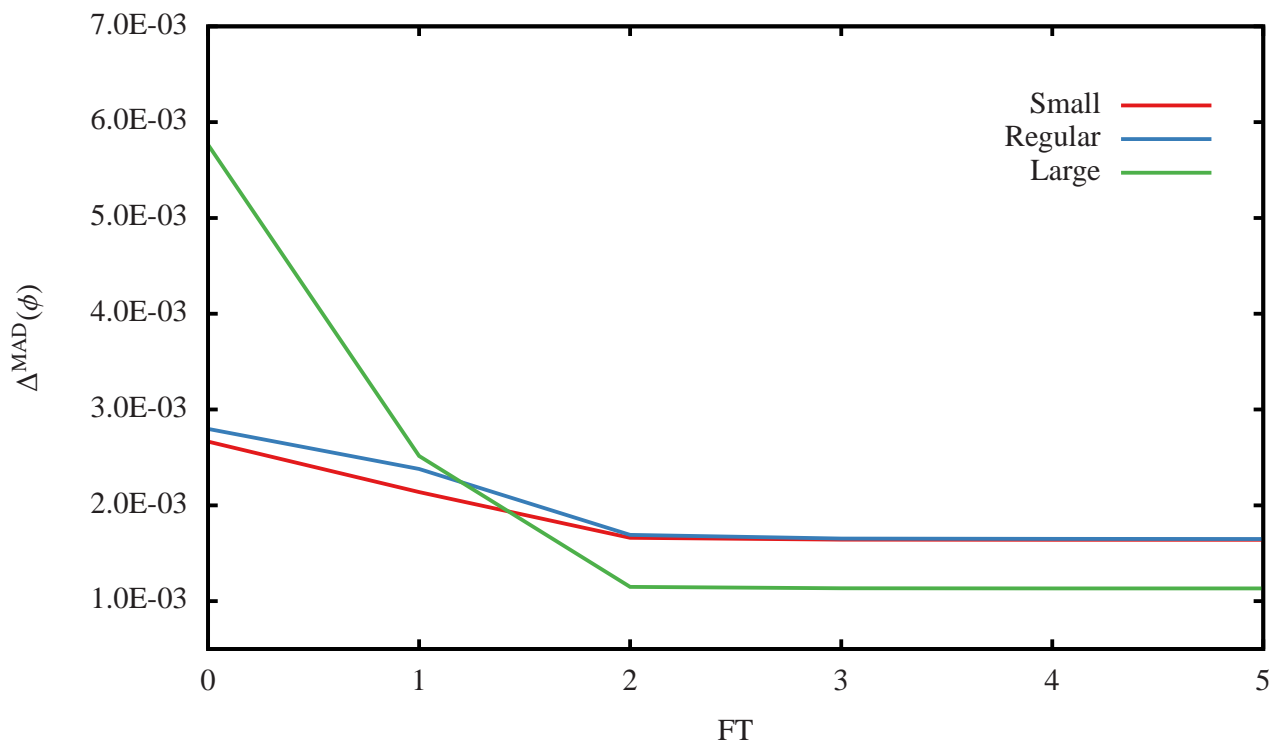

Figure S8: Influence of the capping group type on density and ESP errors in 3-FDE calculations for TC5b in solution over the course of five FT cycles (cycle $0=$ MFCC). Errors are given with respect to supermolecular results obtained with M06-2X (density) or CAMY-B3LYP (ESP). 
Table S2: Density and ESP errors for TC5b in solution obtained in supermolecular calculations with ADF performed with various methods and the DZP basis set. The reference is a cc-pVDZ calculation with the same method carried out with ORCA. The second column gives errors obtained with the default COSMO settings of ADF (identical to Tabs. I (b) and S1 (b)), while these settings have been adjusted to match the TuRBoMoLE defaults for the results presented in the third column.

(a) Density error $\left[\Delta^{\mathrm{RMS}}(\rho)\right.$, unitless $]$

Method

Default COSMO settings

BLYP

B3LYP

M06-2X

(b) ESP error $\left[\Delta^{\operatorname{MAD}}(\phi)\right.$, a. u. $]$

Method

Default COSMO settings

BLYP

0.0040

0.0040

0.0041

B3LYP

M06-2X
0.02468

0.02398

0.02270
0.02274

Adapted COSMO settings

0.02472

0.02402

0.0035

0.0035

0.0035 